\title{
Techniques for embedding instrumentation in pressure vessel test articles
}

\author{
Michael Cornelius \\ ATK Thiokol Propulsion, Science and Engineering Huntsville Operations \\ P.O. Box 9033, Huntsville, AL, 35812
}

\begin{abstract}
Many interesting structural and thermal events occur in materials that are housed within a surrounding pressure vessel. In order to measure the environment during these events and explore their causes instrumentation must be installed on or in the material. Transducers can be selected that are small enough to be embedded within the test material but these instruments must interface with an external system in order to apply excitation voltages and output the desired data. The methods for installing the instrumentation and creating an interface are complicated when the material is located in a case or housing containing high pressures and hot gases. Installation techniques for overcoming some of these difficulties were developed while testing a series of small-scale solid propellant and hybrid rocket motors at Marshall Space Flight Center. These techniques have potential applications in other test articles where data are acquired from materials that require containment due to the severe environment encountered during the test process. This severe environment could include high pressure, hot gases, or ionized atmospheres. The development of these techniques, problems encountered, and the lessons learned from the ongoing testing process are summarized.
\end{abstract}

Keywords: Instrumentation, pressure vessel, pressure transducer, thermocouple, heat flux gage, sealing gland

\section{INTRODUCTION}

If ideal measuring devices were applied to an experiment perfect data could be acquired. An ideal measuring device would occupy no space, have no volume, and would not remove any energy from the medium that it was measuring or change the measured parameter in any way. This ideal device would convert the energy being measured into digital data without any losses and the data curve would be a flawless representation of the actual event. Of course this ideal transducer would not require any excitation voltage or signal conditioning and would transmit the information without the benefit of wiring or transmitter. The data would be accurate to infinite precision, and no analyst would ever dare question it validity. It can only be assumed that perfect technicians supervised by perfect engineers would install these devices. But, in the absence of such perfection, the real world of measurement engineering requires some consideration of the less than ideal conditions when selecting and installing instrumentation. These harsh realities are much more apparent and unforgiving when the environment required by the testing includes conditions which must be contained by a protective vessel. Obviously the environment enclosed by this vessel must be safely penetrated so power can be provided to the instruments and data can be transmitted back from the devices. The transducer and its associated wiring must be installed using techniques that enable them to successfully exist within this environment for the required test duration. It is under these conditions that even simple and straightforward applications become complicated.

\section{METHODOLOGY}

\subsection{Internal solid and hybrid rocket motor environment}

The environment encountered in the interior of a solid or hybrid rocket motor includes extremely hot gases that can exceed 5,000 degrees Fahrenheit. These gases are also very abrasive because they contain aluminum particles, oxidizing compounds and have become ionized by the extreme heat. The pressure inside the rocket motor changes from ambient just before ignition and ramps to several hundred psi a few hundredths of a second. Layers of composites, insulators, and a metal or composite case contain these harsh conditions. Many of the test parameters which require instrumentation are buried several layers into the rocket motor materials and require careful installation of the instrumentation and wiring harness to prevent the escape of hot gas which would destroy the validity of the test if not the entire test article. Because 
of the cost involved in testing large-scale solid rocket motors, preliminary testing for any design or material change is usually done with a much less expensive subscale test article. These subscale test articles are designed to mimic one or more of the actual parameters encountered on the full-scale hardware. By varying the test parameters the performance and limits of the new design or material can be explored. If a series of test motors is fired with the same basic parameters then the repeatability and reliability of the test data can be determined.

\subsection{Measuring devices}

Many of the challenges encountered during the installation of instrumentation in a solid rocket motor might be applied to other disciplines. A simple way of thinking about a solid or hybrid rocket motor is to consider it a pressure vessel, which must contain an event that consists of a very short initiation transient followed by several seconds of extreme heat and pressure. Many other test apparatus including pressure vessels with various internal environments that must be fully contained might benefit from the installation techniques developed during the static tests of solid rocket motors. Any device that requires connection to external power or data systems using conventional wiring could be installed using some variation of these techniques. These techniques were developed during several test series over a period of several years. The conventional instrumentation installed included miniature piezo pressure transducers, $\mathrm{K}, \mathrm{S}$, and $\mathrm{C}$ type thermocouples, strain gages, and heat flux gages. The sensors used during this test series were, for the most part, commercial off the shelf instrumentation. Criteria used for the instrumentation selection included small physical size, reasonable accuracy, and the capability of being embedded inside a test article surrounded by a pressure vessel. Data from these miniature instruments were acquired using conventional external data acquisition systems with appropriate data rates, accuracy, and resolution. The connection between the internal instrumentation and the external data system was made using small gage solid copper or thermocouple wires with polyimide coatings. Additional electrical insulation was added as needed and more robust extension wires were installed to the electrical circuits after they exited the pressure vessel.

\section{RESULTS}

\subsection{Pressure transducer installation techniques}

The pressure transducers installed in this test series were almost exclusively miniature piezo or silicon strain gage transducers. The transducers have a small diaphragm on a threaded extension attached to a miniature electronic package (See Figure 1). The transducers selected were ranged for absolute pressure (PSIA) and did not require the exposure to atmospheric pressure for reference.

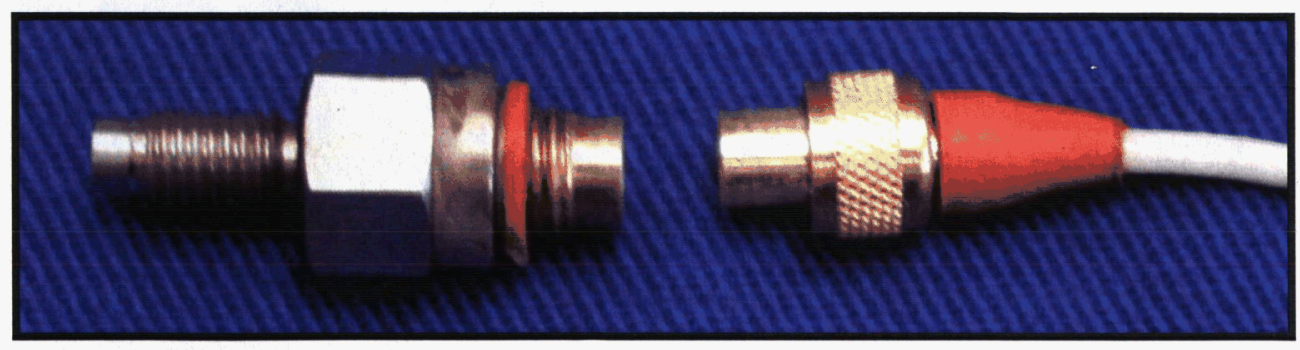

Figure 1 Miniature pressure transducer and mating cable

An adapter was used to direct the gas being measured back to the face of the diaphragm. This adapter was critical to the success of making internal pressure measurements and consisted of a small pickup tube silver soldered to a fitting with threads to match the threads of the transducer (See Figure 2). The adapter provided three important functions. First the adapter allowed the transducer to be installed some small distance away from the hot gases. This allowed the gases to stagnate and cool in the pickup tube and not over-heat the transducer. The second advantage gained from the adapter is that, because the tube was considerably smaller in diameter, it significantly decreased the surface area needed in the test region to acquire this measurement. The final advantage gained from the adapter was the ability to securely bond the adapter in place with a custom installation. The pickup tubes were fabricated longer than needed for any one installation and were then fitted and custom cut for each location. This created the opportunity for a large area of adhesive around the adapter and resulted in a torturous gas path with several 90-degree turns around the shoulder to restrict any gas escaping from the test article. The pressure transducers were selected with miniature connectors that allowed them to be 
installed without the entanglement of hookup wire. The connecting cable was then installed after the adapter was bonded in place.

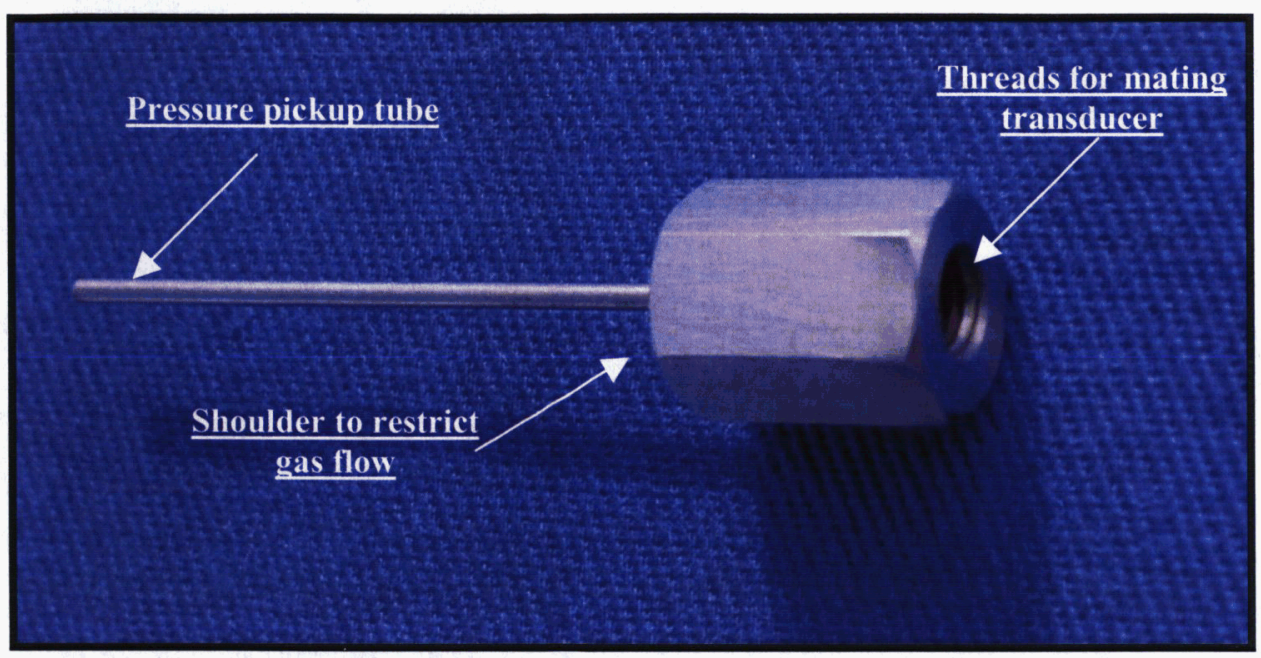

Figure 2 Pressure transducer adapter

An early motor design allowed the hookup cable to be routed out the pressure vessel and sealed with RTV. This design, while overall a successful one, allowed for the possibility of small gas paths along the wire harness and resulted in several minor leaks. Later designs required an internal conversion from the stranded multi-conductor hookup cable to individual solid conductors (See Figure 3). The solid hookup wires were typically 30 AWG with polyimide coating. These small gauge wires allowed the routing paths to be minimized and allowed a higher wire count in the wire feedthroughs. The feedthroughs required the use of a solid wire to allow the sealing gland to compress against. Damage to the conductors occurred when an attempt was made to use stranded wires.

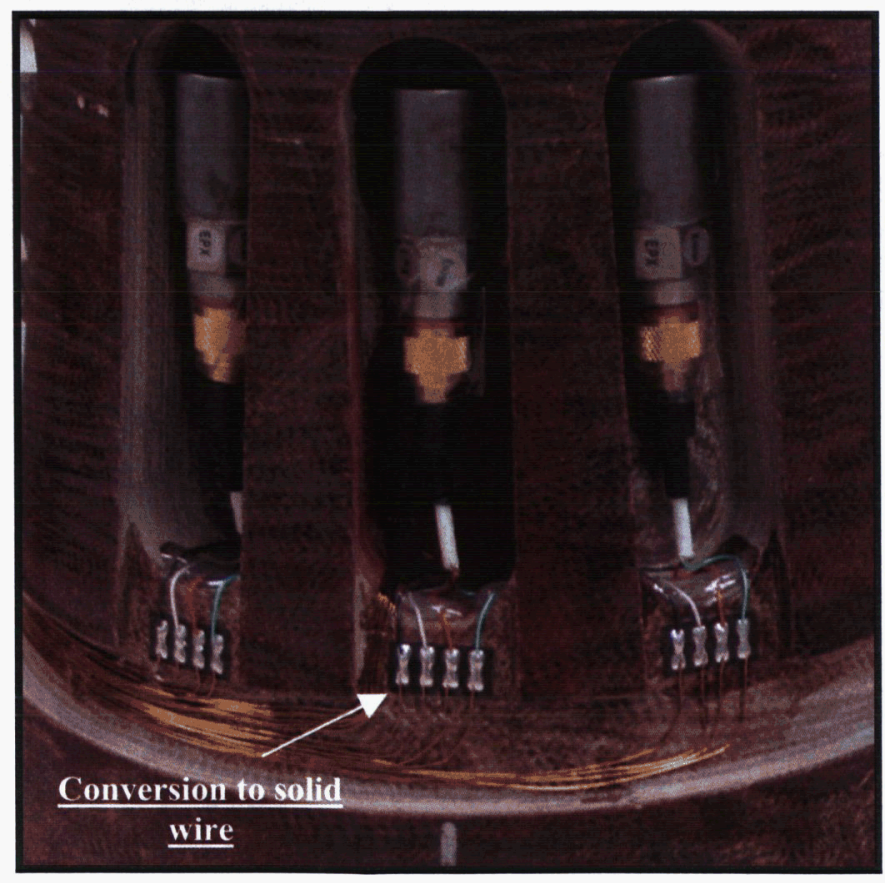

Figure 3 Stranded to solid wire conversion and routing 


\subsection{Thermocouple installation techniques}

Thermocouples were exclusively used to acquire temperature data during these tests. The simplicity, ruggedness, and wide temperature range made them ideal for this application. Early installations of thermocouples were miniature sheath type devices. The sheath diameter of these Chromel-Alumel (K-type) or Platinum-Rhodium (S-type) thermocouple was 0.010-inch. The sheath material was typically Inconel or Platinum, and the thermocouple tips were ungrounded.

During the development of the instrumentation for this test series, it was discovered that the sheath-type thermocouple design was not the optimal installation for an accurate measurement of hot gases. The sheath itself created additional errors in the temperature measurement by reflecting radiated heat and insulating portions that conduct heat away from the thermocouple bead before it responded to the change in temperature. A new approach to thermocouple installation resulted in suspending a bare wire thermocouple in the test article using ceramic tubes as stand offs (See Figure 4). The thermocouples were butt-welded to create a thermocouple with the wires extending from the bead in opposite directions. This allowed the thermocouple to be suspended in the gas path with the wires routed out of separate ceramic tubes. Ktype thermocouples were locally fabricated using this technique. New test requirements included higher temperature ranges, so Tungsten-Rhenium (C-type) thermocouples were added to the test articles and were custom fabricated by outside vendors. Because the C-type thermocouples were very stiff and brittle, a transition from thermocouple grade to extension grade wire was made as soon as feasible. The transition between the two wire types proved to be a weak point with a potential for broken gages. This problem was resolved by careful handling of the thermocouples and by designing the thermocouples so the transition was made inside the ceramic stand off tube. This design allowed the ceramic tube to provide additional support to the weaker junction. The final configuration included 0.005 -inch thermocouple wire with 0.020 -inch diameter extension wire. The suspended thermocouple design required $90^{\circ}$ bends in the wire on either side of the thermocouple bead. Large $(0.010$-inch) and smaller $(0.003$-inch) thermocouple wires were also experimented with. The larger wires were difficult to work with and resulted in more broken thermocouple beads than the 0.005 -inch wire. The smaller wire proved to be too fragile for processing and installation. Because this new installation required bare wires, the conductors were isolated from each other and from the conductive test article using non-conductive adhesives and small fiberglass tubing.

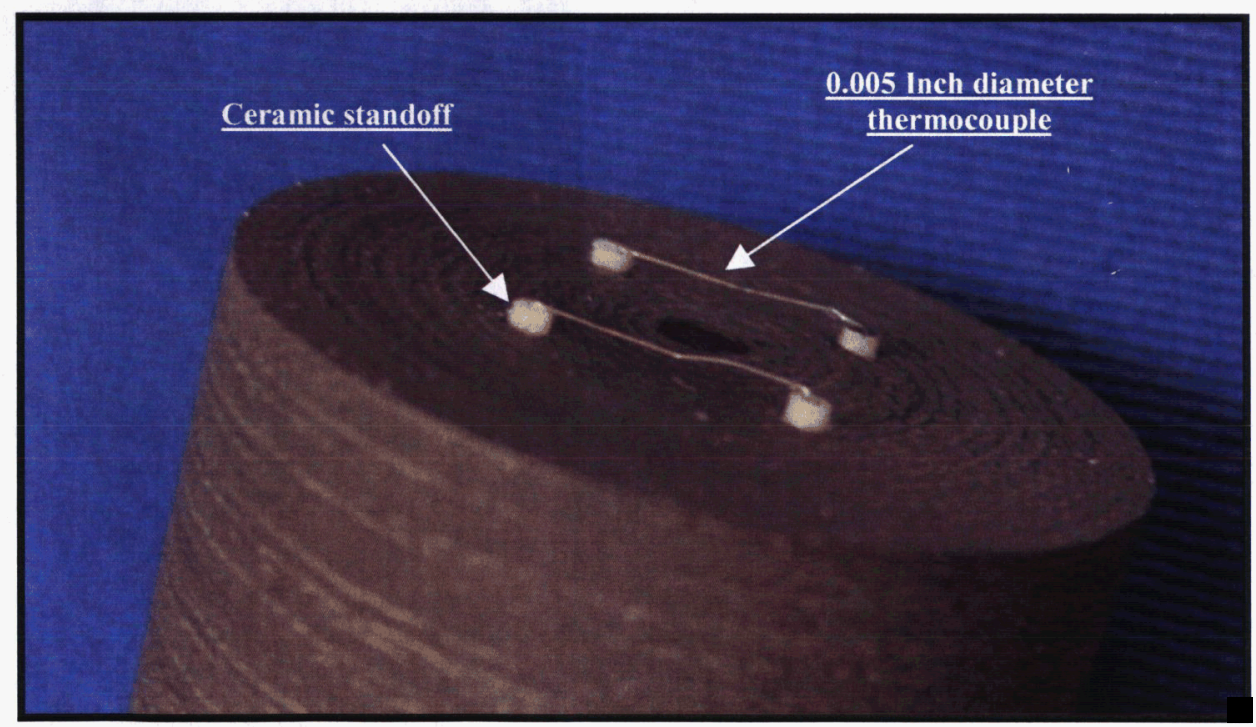

Figure 4 Suspended C-type thermocouple

\subsection{Heat flux gage installation}

Several attempts were made to obtain heat flux data from the hot gases found inside the solid rocket motor with both commercial and custom built transducers. The commercial gages were primarily miniature hot walled Gardon gages, and the custom-built gages were of the slug calorimeter design. Because of the nature of the hot gas environment, sealing around the heat flux gage was an important consideration. Commercial gage designs with a built-in shoulder between the gage body and the sensing element were selected whenever possible. This allowed the transducer to be 
installed with a technique similar to that used with the pressure adapters. The face of the gage installed in the gas path was much smaller in diameter than the remaining transducer body. This created the opportunity for a sufficient adhesive bond and created a natural barrier for any gas that might leak past the bond line. The majority of heat flux gages were built with 30 AWG (0.010-inch diameter) solid hookup wires for the same routing and gas path considerations used on other instruments. A limitation of the hot wall Gardon gage is the loss of reliable data when the body of the transducer exceeds $400^{\circ} \mathrm{F}$. In order to determine when this event occurred a K-type (Chromel-Alumel) thermocouple was incorporated into the design or added to the instrument when possible. Custom-built slug-type calorimeters were also fabricated and installed. These gages were typically a simple cylindrical or rectangular mass of graphite with one or more thermocouples installed in the center of the mass to measure the temperature gradient. The heat flux could be determined analytically using the temperature gradient data along with the thermal conductivity and mass of the slug material.

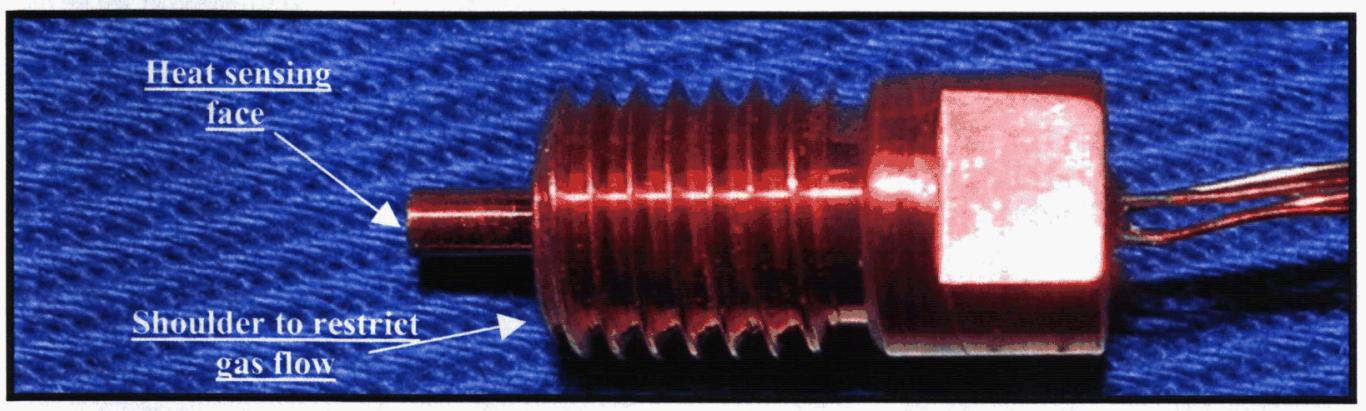

Figure 5 Miniature Commercial Hot Wall Heat Flux Gage

\subsection{Wire feed throughs}

A great deal of thought and effort went into designing and installing the instrumentation using techniques to minimize, if not eliminate, potential leak paths around the instrumentation. The creation of a gas path in a severe environment, like that found in a solid rocket motor, could easily result in the loss of data, instrumentation, and in extreme cases the loss of the pressure vessel. If the leak paths can be restricted to small areas and any mass flow minimized it has been shown that the hot gas will stagnate and cool very quickly. As the final safety measure the use of a pressure seal around the instrumentation wires where they exit the pressure vessel was used. When designing the instrumentation this seal was usually intended to become a secondary seal to trap and stagnate any gas flow caused by leaks around the instrumentation. The exit port for the instrumentation wires was often back filled with RTV, polysulfide, or an adhesive, during the installation process. Because the sealing gland could not effective seal around stranded wires, solid wires were used for all of the instrumentation. Instruments with solid wires were specified when possible, but if the instrumentation interface was manufactured with stranded wires a solder tab was installed internally in the pressure vessel to allow for conversion to solid wires (See Figure 4).

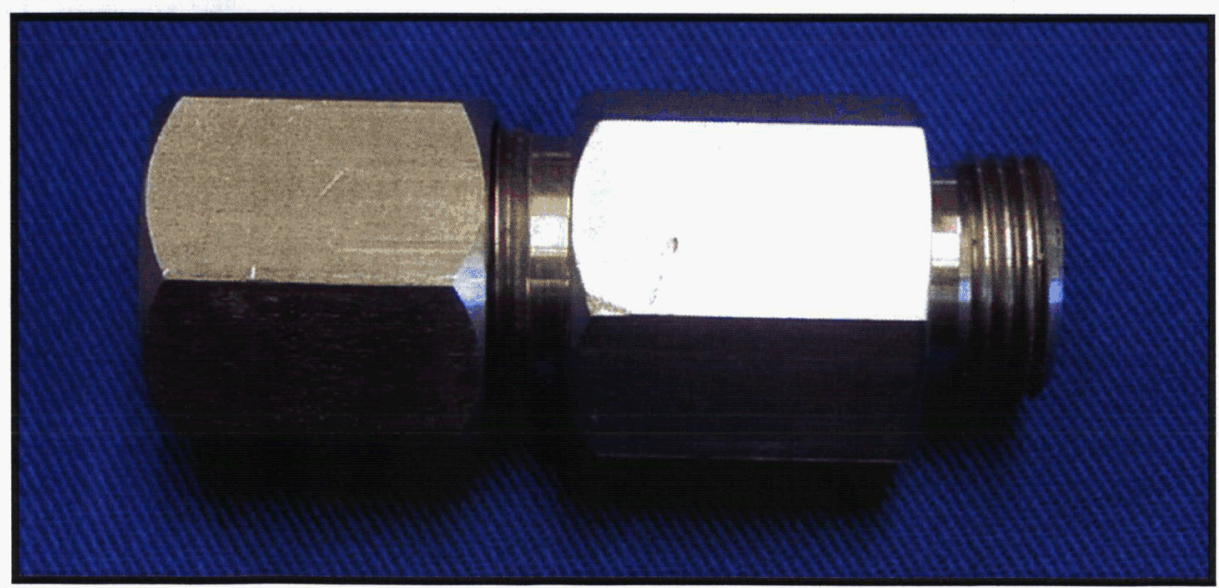

Figure 6 Assembled pressure gland 
Commercial feedthroughs were purchased for this test series and were often custom-fabricated for a particular application (See Figure 6). Two multi-conductor pressure gland designs were used during this test series. One design isolated the wires with internal ceramic parts and was used whenever possible because the ceramic was electrically nonconductive (See Figure 7). This decreased the probability of scraping the polyimide coating on the wires and creating a short. The wire count on several test motors was higher than could be accommodated by a sealing gland with internal ceramic isolators. This problem was resolved by utilizing a high-capacity sealing gland with internal metal components, which allowed custom holes to be drilled by the manufacturer (See Figure 8). The number and diameter of the holes were determined by the test article and instrumentation requirements.

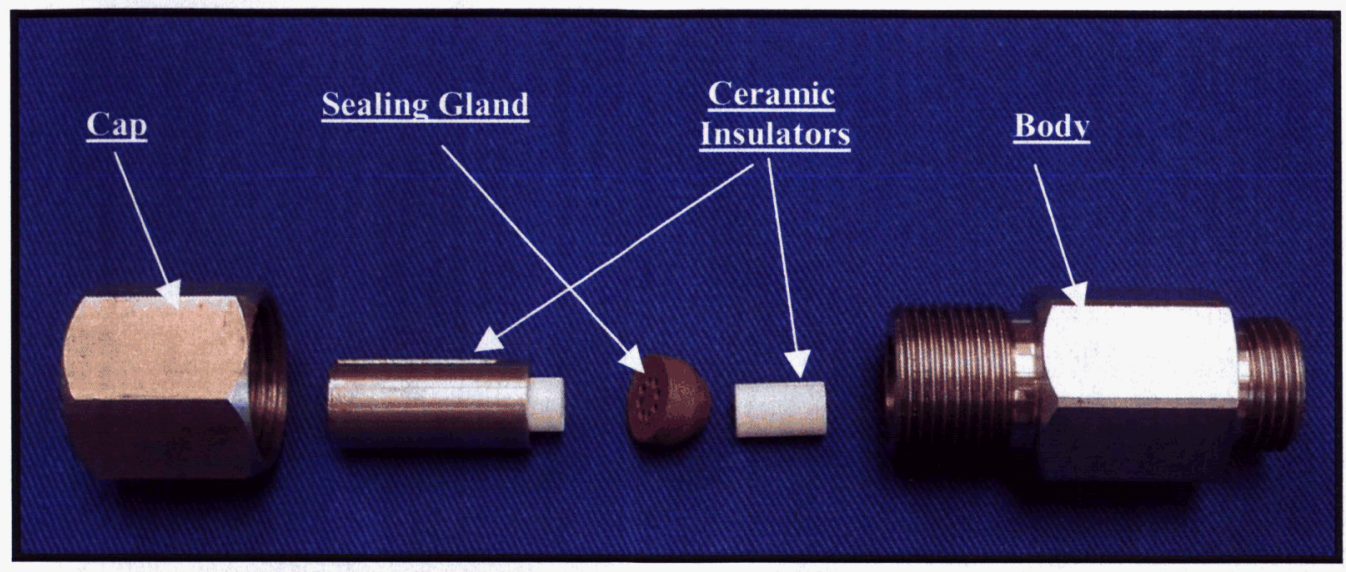

Figure 7 Pressure gland with ceramic insulator

Several problems were encountered, and eventually solved, when utilizing high density sealing glands with small diameter wire. The problems revolved around the need to perfect installation techniques and were not attributed to the sealing gland design or the manufacturer. The primary problem encountered was shorting of instrumentation wires to the pressure vessel due to slack in the wiring when assembling the internal parts. This problem was more apparent with the custom-built glands because of the internal metal components and the higher wire density utilized when using a custommade fitting with a maximum number of feedthrough holes. This design consists of a metal body threaded for the appropriate pressure port with an internal sealant gland that is compressed between a seat and a follower. These internal parts have the correct number of through holes with a diameter sized for the wire gage. A cap over the entire fitting is torqued against the follower to provide the force required to seal the gland.

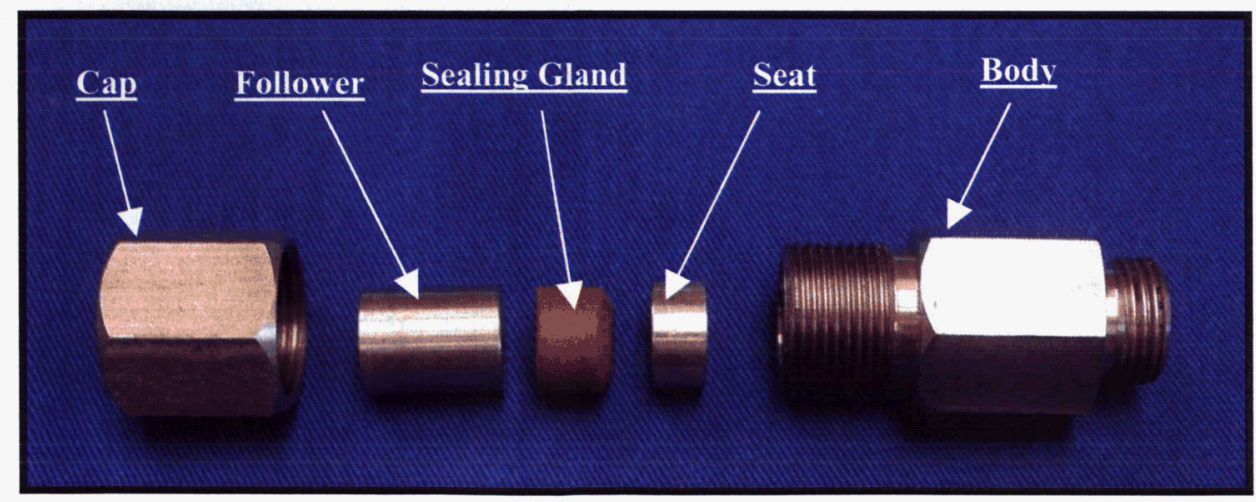

Figure 8 Pressure gland with metal seat and follower

Problems where encountered when sealing twenty or more 30 AWG wires in the same fitting. The typical installation required passing the small gage wires through the body of the fitting that is usually already installed in the pressure port. 
The seat, sealant, and follower are then staged on a work surface close to the pressure vessel and the wires are passed through the feed through holes in the internal parts (See Figure 9). After all the wires have been routed through each internal part, they were then installed into the body of the sealing gland prior to being torqued.

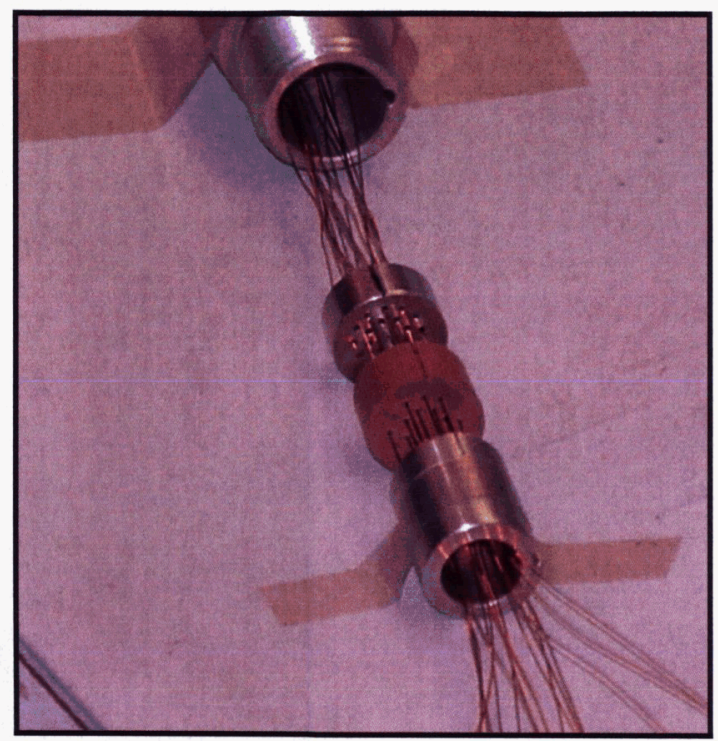

Figure 9 Pressure gland assembly process

The shorting problems that were encountered were created when slack in the small gage wires allowed them to bunch up in front of the seat and become trapped between the front edge of the seat and the body of the sealing gland. The wires were crushed and shorted to the pressure vessel through the body of the fitting when force was applied to the cap and the sealant was compressed (See Figure 10). This problem was not apparent during the assembly process; and because continuity, or lack of continuity, between the conductors and the pressure vessel was not measured prior to testing, the shorted wires resulted in loss of test data.

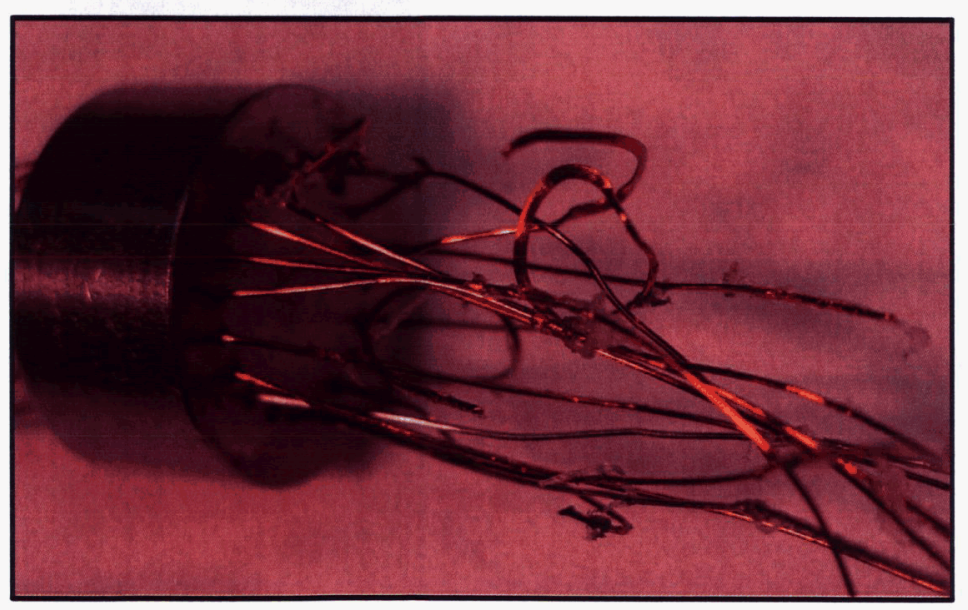

Figure 10 Wire damage in pressure gland 
The instrumentation installation was modified to help eliminate this problem by bonding the wires inside the test article so the slack in the conductors could be pulled out during the pressure gland assembly. It was also observed that the wires shorted out to the body were not as long as the wires that were not shorted because some wire length was trapped between the seat and the body. Subsequent installation procedures included marking the wire length or cutting all the wires the same length to provide a visual indication of a potential problem. When the problem is uncovered prior to applying torque, the slack in from the seat can be pulled out, and the shorting situation can be avoided. This new assembly technique required two people for the process. One person would assembly the parts while the second person maintained tension on the wires and verified the reference marking on the wires did not move during the assembly.

\section{CONCLUSIONS}

The installation of instrumentation in a pressure vessel containing an extreme test environment can be very challenging. The challenges that extend beyond the typical instrumentation installation include sealing against the internal environment and providing an electrical path that allows excitation voltages to be applied to the transducers and the acquisition of the data from the transducers. Many of these challenges were overcome during a series of static tests on small-scale solid and hybrid rocket motors. Application of the techniques developed and the lessons learned are not restricted to rocket motor testing but can be used when acquiring data on other test programs where extreme environments must be contained by a pressure vessel.

\section{ACKNOWLEDGEMENTS}

The development of these techniques and the solution to the problems encountered during the solid rocket motor test series were the result of inputs and suggestions from a group of ATK Thiokol Propulsion employees who are talented technical experts. This instrumentation team included Marcus Kennamer, Jason Parker, Shane Perkins, Terri Robenstine, John Swartz, and Tony Newton. The testing reported would not have been a success without their dedication and support. 